\title{
Development modeling methods of analysis and synthesis of fingerprint deformations images
}

\author{
Haider Hassan Majeed Al Karaawi ${ }^{1}$, Mohammed Qasim Dhahir ${ }^{2}$, Ibrahim Ahmed Alameri ${ }^{3}$ \\ ${ }^{1}$ University of Kerbala, Iraq \\ ${ }^{2}$ Department of English Language, University of Kufa, Iraq \\ ${ }^{3}$ Jaber ibn Hayyan Medical University, Iraq \\ ${ }^{3}$ Faculty of Economics and Administration, University of Pardubice, Czech Republic \\ ${ }^{1,2,3}$ Ministry of Higher Education and Scientific Research, Iraq
}

\begin{tabular}{l} 
Article Info \\
\hline Article history: \\
Received Mar 8, 2020 \\
Revised May 14, 2020 \\
Accepted May 26, 2020 \\
\hline Keywords: \\
Correlation \\
Dactyloscopy \\
Deformations \\
Heterogeneity \\
Minimum spanning tree (MST) \\
Orthonormality \\
Papillary
\end{tabular}

\section{Corresponding Author:}

Mowafak K. Mohsen,

Ministry of Higher Education and Scientific Research,

University of Kerbala,

Kerbala, Iraq.

Email: mowafak.k.m@gmail.com

\begin{abstract}
The current study is to develop modeling methods, analysis and synthesis of fingerprints deformations images and their application in problems of automatic fingerprint identification. In the introduction justified urgency of the problem, is given a brief description of thematic publications. In this study will review of modern technologies of biometric technologies and methods of biometric identification, the review of fingerprint identification systems, investigate for distorting factors. The influence of deformations is singled out, the causes of deformation of fingerprints are analyzed. The review of modern ways of the account and modeling of deformations in problems of automatic fingerprint identification is given. The scientific novelty of the work is the development of information technologies for the analysis and synthesis of deformations of fingerprint images. The practical value of the work in the application of the developed methods, algorithms and information technologies in fingerprints identification systems. In addition, it has been found that our paper "devoted to research methods and synthesis of the fingerprint deformations" is a more appropriate choice than other papers.
\end{abstract}

Copyright (C) 2020 Institute of Advanced Engineering and Science. All rights reserved.

\section{INTRODUCTION}

Currently, biometric (using the physical and behavioral characteristics of a person) [1,2] personality identification technologies and systems based on them are widely used in various fields [3, 4]: from access to premises to electronic commerce and government systems for various purposes. The most common biometric identification method is fingerprint identification [5-7]. Despite numerous studies in the field of fingerprinting conducted by such scientists, the accuracy of fingerprint identification has not reached its potential. The main reason is the complexity of modeling, accounting and compensation of numerous distorting factors, among which are noise, small areas of intersection of fingerprints presented for comparison, and elastic deformations.

The most important applications of fingerprint deformation models are the synthesis of artificial finger applications to simulate technological tests in civil identification systems, and a priori elimination of deformation by calculating the "non-deformed" state of a fingerprint for a variety of applications at the registration stage. Deformations are one of the strongest and least studied factors. In this regard, the topics related to the study of deformation of fingerprints is relevant. For achievement of the goals need a solution of the tasks, some development of mathematical model for processing of fingerprint deformation have been conducted, i.e: development of methods for analyzing of fingerprint deformities images [8], statistical analysis 
of character and the structure of deformations; development of methods for the synthesis of fingerprint deformities images [9], development of the modeling algorithm for the fingerprint deformation [10], and development of a priori registration algorithm for the fingerprint deformation [11]. In this paper, modern technologies of biometric technologies, methods of biometric identification and fingerprint identification systems will be reviewed; distorting factors will be investigated; and deformation of fingerprints will be analyzed.

\section{RESEARCH METHOD}

This work uses modern methods of image processing and image recognition [12, 13], probability theory and mathematical statistics [14], theory of elasticity, methods of programming and computer simulation [15]. The deformation of the fingerprint image is determined by a displacement map, which can be interpreted as displaying one image to another image:

$$
f=\operatorname{Im}_{1} \rightarrow \operatorname{Im}_{2}
$$

where $\mathbf{I m}_{1}, \mathbf{I m}_{2}$ - images of fingerprints.

Next, we use the representation of the map (1) in the form of a displacement map $\mathbf{u}$. The action of the mapping $\boldsymbol{f}_{\mathbf{u}}$, associated with displacements $\mathbf{u}$, can be represented by the following expression:

$$
\begin{aligned}
& \operatorname{Im}_{1}(x, y)=F_{u}^{-1}\left(\operatorname{Im}_{2}(x, y)\right)= \\
& =\frac{1}{\operatorname{det} J_{u}(x, y)} \operatorname{Im}_{2}\left(x+u_{x}(x, y), y+u_{y}(x, y)\right) \\
& J_{u}(x, y)=\left(\begin{array}{cc}
1+\frac{\partial u_{x}}{\partial x} & \frac{\partial u_{x}}{\partial y} \\
\frac{\partial u_{y}}{\partial x} & 1+\frac{\partial u_{y}}{\partial y}
\end{array}\right)
\end{aligned}
$$

In dynamics as shown in Figure 1, we can assume that $\mathbf{u}$ it depends on time. Suppose that a fingerprint is an elastic object. Then its dynamics is described by the following system of equations of the theory of elasticity:

$$
\begin{aligned}
& \frac{\partial \sigma_{x y}}{\partial y}+\frac{\partial \sigma_{x x}}{\partial x}+f_{x}=\rho \frac{\partial^{2} u_{x}}{\partial t^{2}} ; \frac{\partial \sigma_{x y}}{\partial x}+\frac{\partial \sigma_{y y}}{\partial y}+f_{y}=\rho \frac{\partial^{2} u_{y}}{\partial t^{2}} \\
& \varepsilon_{x x}=\frac{\partial u_{x}}{\partial x} ; \varepsilon_{y y}=\frac{\partial u_{y}}{\partial y} ; \varepsilon_{x y}=\varepsilon_{y x}=\frac{1}{2}\left(\frac{\partial u_{x}}{\partial y}+\frac{\partial u_{y}}{\partial x}\right)
\end{aligned}
$$

where $\mathbf{u}_{\mathbf{x}}$ and $\mathbf{u}_{\mathbf{y}}$ - components of the displacement map along the $\mathrm{x}$ and $\mathrm{y}$ axes respectively, - acting external forces, $\boldsymbol{\varepsilon}$ - tensor of $f=\left(f_{x}, f_{y}\right)$ tension, $\boldsymbol{\sigma}$ - pressure tensor, $\boldsymbol{\rho}$ the density. Components of tensors $\boldsymbol{\varepsilon}$ and $\boldsymbol{\sigma}$ are related by a linear relationship:

$$
\left(\begin{array}{l}
\partial_{x x} \\
\partial_{y y} \\
\partial_{x y}
\end{array}\right)=\boldsymbol{C}(x, y)\left(\begin{array}{l}
\varepsilon_{x x} \\
\varepsilon_{y y} \\
\varepsilon_{x y}
\end{array}\right)
$$

Solutions of equations are determined by the values of the elasticity matrix $C(x, y)$. The matrix is unstable, since the surface of the finger is not uniform in mechanical properties. Heterogeneity is associated with various mechanisms of deformation under the action of forces along and across the direction of the papillary pattern [16], as shown in Figure 2. In the coordinates associated with the local direction of the papillary pattern, matrix $\boldsymbol{C}^{\epsilon}$ has four degrees of freedom and is equal to 1:

$$
\boldsymbol{C}^{\epsilon}(z, w)=\left(\begin{array}{ccc}
E_{z}^{-1} & -v_{z w} E_{w}^{-1} & 0 \\
-v_{w z} E_{z}^{-1} & E_{w}^{-1} & 0 \\
0 & 0 & G
\end{array}\right) ; v_{z w} E_{w}^{-1}=-v_{w z} / E_{z}^{-1}
$$


where $E_{z}, E_{w}$ - modules along and across the direction field, $v_{z w}$ and $v_{w z}$ - Poisson's ratio, $\boldsymbol{G}$ - Shear modulus. When changing to the original global coordinates (6).
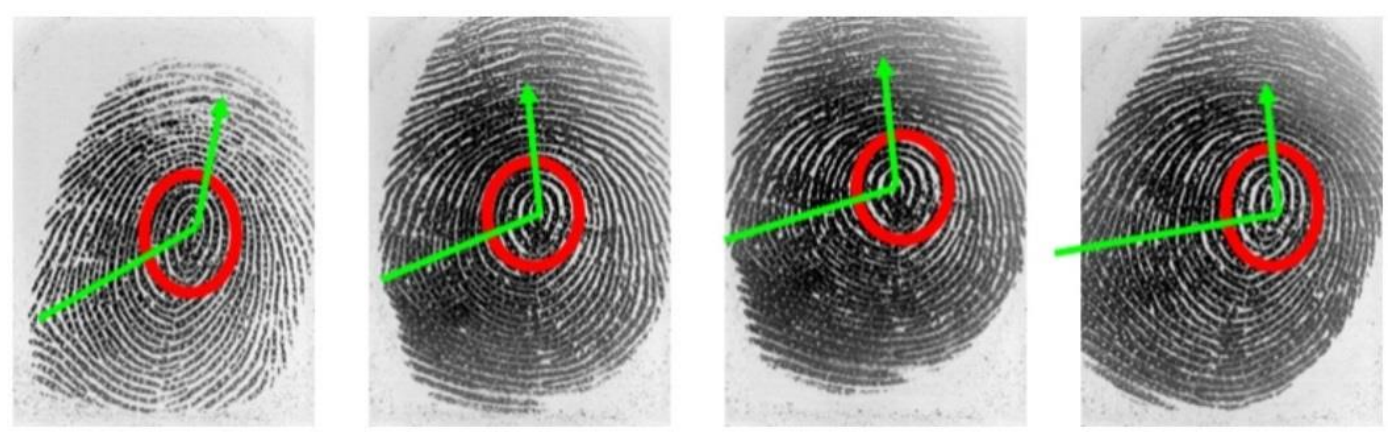

Figure 1. The process of deformation of fingerprints (screencast)
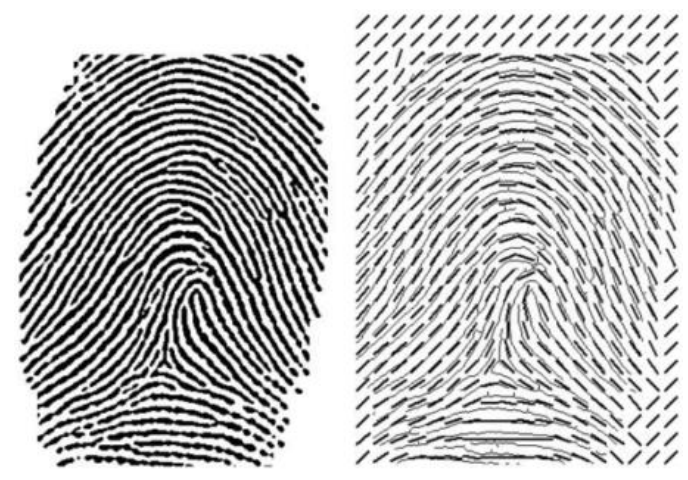

Figure 2. Direction of the flow of papillary lines

\section{RESULTS AND DISCUSSION}

In fingerprint identification the main interest is the moment of image capture $[16,17]$. Usually this happens, when the image is still, the acting forces have already balanced the tension, cause displacement. Such a condition can be written as the following equation

$$
\frac{\partial \sigma_{x y}}{\partial_{y}}+\frac{\partial \sigma_{x x}}{\partial_{x}}+f_{x}=0 ; \frac{\partial \sigma_{x y}}{\partial_{x}}+\frac{\partial \sigma_{y y}}{\partial_{y}}+f_{y}=0 ;
$$

The system of (7) and (4) determines the deformation of the fingerprint. The following difficulties of its direct solution are noted. First, there is no initial state of the print, from which the deformation begins. First, there is no initial state of the print, from which the deformation begins. Deformation is a consequence of the process of two-dimensional scanning of a three-dimensional object see Figure 3. Secondly, the active forces are unknown. There is no engineering way to measure them. From the theory of elasticity, it is known that the solution of (4) and (7) can be sought as a minimum of the following energy function

$$
E=-\iint_{S}\left(u_{x} f_{x}+u_{y} f_{y}\right) d x d y+\frac{1}{2} \iint_{S}\left(\varepsilon_{x x} \sigma_{x x}+\varepsilon_{y y} \sigma_{y y}+\varepsilon_{x y} \sigma_{x y}\right) d x d y
$$

In connection with the above-mentioned problem of the inability to estimate the effective forces, the negative term in (8) also cannot be directly evaluated. For its elimination, a method of approximate deformation calculation is proposed. For two random images of one fingerprint, will transforming one into another by deformation, you can find corresponding to each other control points of the image as shown in Figure 4, which gives information on the general direction of the acting forces. 


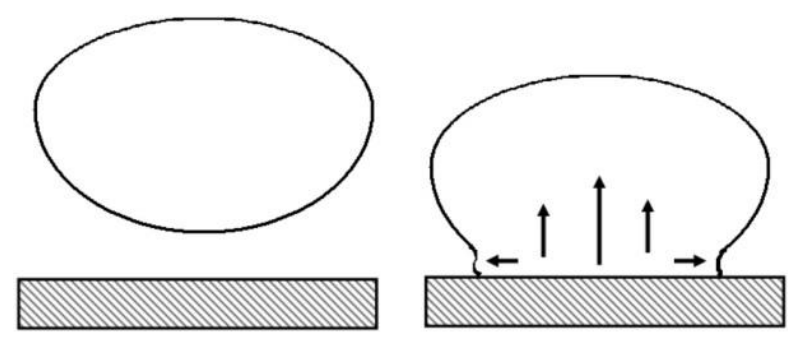

Figure 3. The appearance of deformation of fingerprints

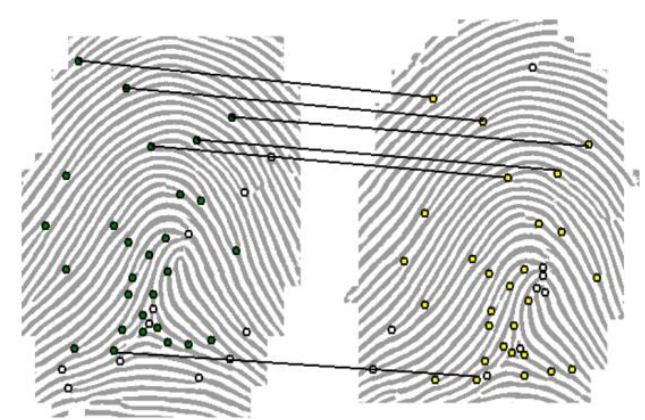

Figure 4. Matching fingerprint checkpoints

The sets of pairs of points corresponding to each other are denoted by $\left\{\boldsymbol{p}_{i}\right\}_{i=1}^{m}$ and $\left\{\boldsymbol{q}_{i}\right\}_{i=1}^{m}$ respectively. As a measure of proximity of two sets of control points, the mean square error

$$
L^{2}(\boldsymbol{u})=\frac{1}{m} \sum_{i=1}^{m}\left\|\boldsymbol{p}_{i}+\boldsymbol{u}\left(\boldsymbol{p}_{i}\right)-\boldsymbol{q}_{i}\right\|^{2}
$$

The functional (9) is qualitatively correlated with the work of the acting forces of the energy functional (8). If a control point shift occurs, it is assumed that external forces act in the direction of displacement. In addition, it is also suggested that the directions of the fluxes of the papillary lines at the control points match with the following functional:

$$
L_{n}^{2}(\boldsymbol{u})=\frac{1}{m} \sum_{i=1}^{m}\left\|\boldsymbol{n}\left(\boldsymbol{p}_{i}+\boldsymbol{u}\left(\boldsymbol{p}_{i}\right)\right)-\boldsymbol{n}\left(\boldsymbol{q}_{i}\right)\right\|^{2}
$$

where $\mathbf{n}$ - functional of taking the normal to the local flow of papillary lines. With allowance for (9) and (10), it is suggested to seek the approximate solution (8) as a minimum of the following functional, consisting of the internal energy of deformation and regularizing the corrections to the discrepancy:

$$
D(\boldsymbol{u})=E_{d}(\boldsymbol{u})+a_{1} L^{2}(\boldsymbol{u})+a_{2} L_{n}^{2}(\boldsymbol{u})
$$

where $a_{1}, a_{2}$ - weight coefficients, internal strain energy $E_{d}$ is calculated by the formula.

$$
E_{d}=\frac{1}{2} \iint_{S}\left(\varepsilon_{x x} \sigma_{x x}+\varepsilon_{y y} \sigma_{y y}+\varepsilon_{x y} \sigma_{x y}\right) d x d y
$$

The minimum of the functional (11) is sought numerically by the finite element method. The displacement map is specified by values in a rectangular grid. For points within a grid element, the values $\mathbf{u}$ are interpolated by bilinear splines. With such interpolation (11) is a quadratic functional of the values at the lattice sites. Thus, the minimum is sought from the condition that all partial derivatives vanish. This condition is a linear equation for the values $\mathbf{u}$ in the lattice sites. The developed algorithm of the solution quickly converges. On average, the calculation of the relative deformation takes less than $0.1 \mathrm{~s}$. Examples of the results of deformation calculations are shown in Figure 5. From the point of view of the research tasks, the optimal result is to find a deformation that translates one image of the print into another image of the same 
print. Therefore, as one of the estimates of the accuracy of deformation calculation, direct overlap is used $\mathbf{I m}_{a v}$ images:

$$
\boldsymbol{I m}_{a v}=\frac{1}{2}\left(\boldsymbol{I m}_{2}+f_{u}\left(\boldsymbol{I m}_{1}\right)\right)
$$

Examples of superposition with allowance for the deformation optimal for (11) in comparison with the optimal motion are shown in Figure 5. The contrast part of the results of superposition indicates the resonance of the papillary lines. The gray part corresponds to inaccurate guidance. It can be seen from the figure that the proposed model allows the image of fingerprints to be accurately converted into each other with high accuracy. There are no edge effects characteristic of empirical deformation models based on a thin metal plate 2 or the Cappelli et al., 3 model. Also, the advantages of the model include the possibility of calculating the relative strain for arbitrary dactyloscopy images for which the corresponding reference points can be found, and for presenting various fingerprints [18].

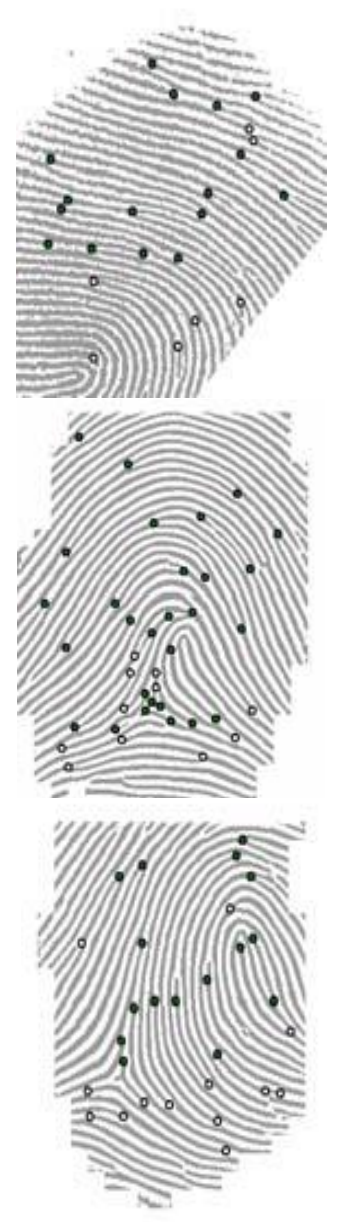

(a)
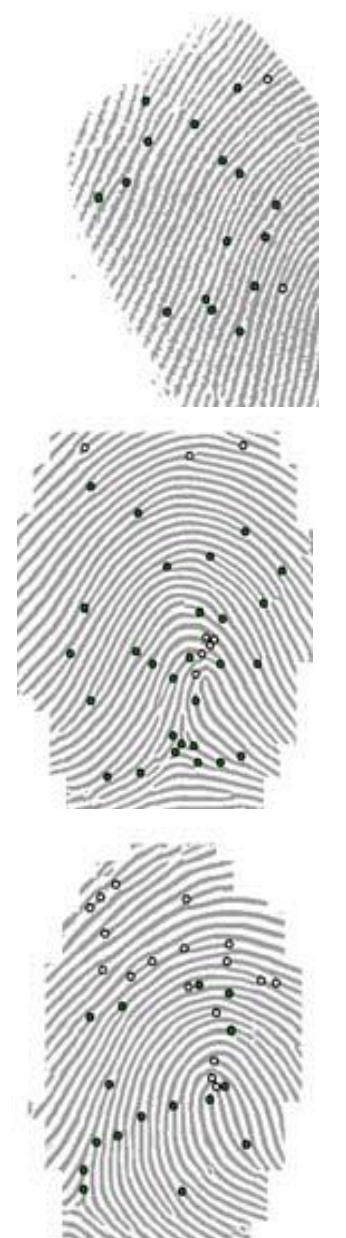

(b)
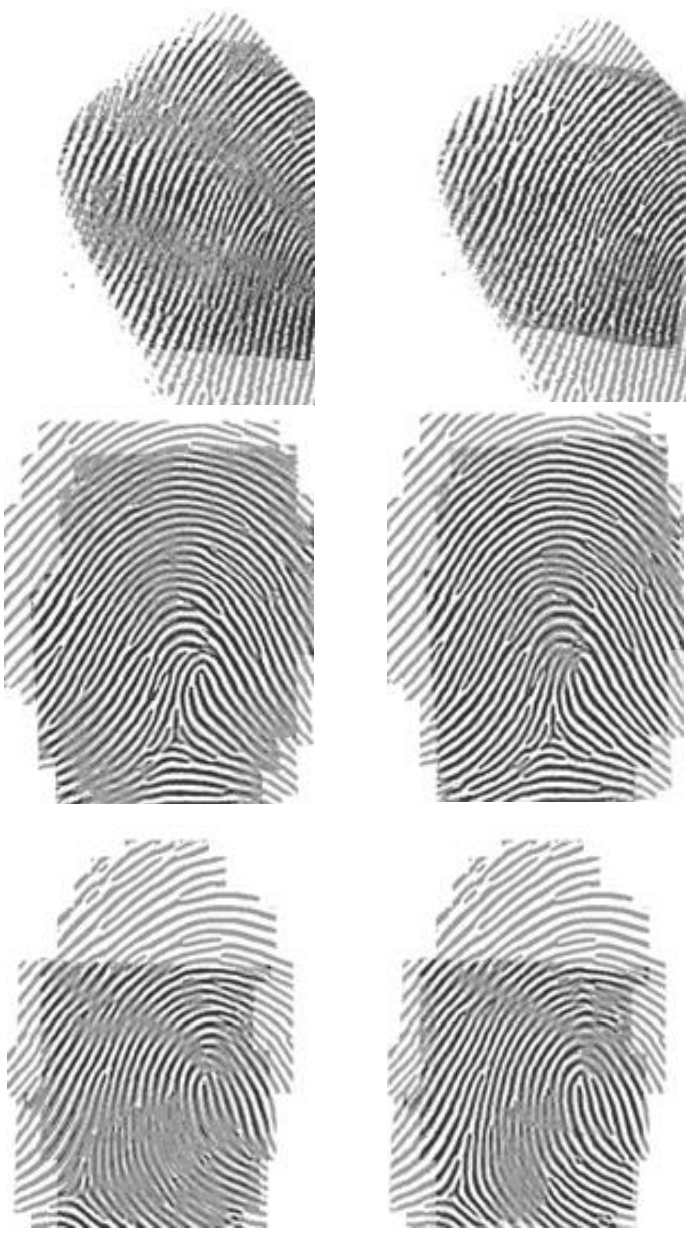

(c)

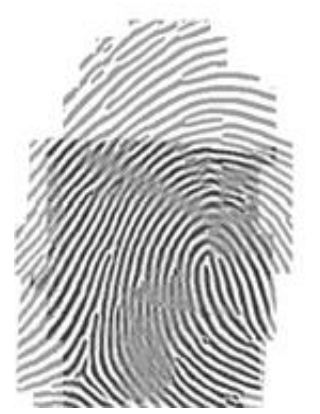

(d)

Figure 5. Example of calculation of the deformation (a), (b) two images of the print, (c) direct overlap when fitting by hard motion, (d) direct overlap after fitting the deformation

The conducted statistical analysis also confirms that the proposed elastic model significantly improves the accuracy of image guidance in comparison with known models. As an objective criterion for the accuracy of modeling the process of deformation of fingerprints, the direct imposition (correlation) coefficient for binarized fingerprint images is used [19]. DET curves of the coefficient of direct overlap for the base FVC2002DB1 (moderate deformation) and FVC2004DB1 (significant deformations) are shown in Figure 6. As can be seen from the figure, the proposed elastic model significantly improves the imposition of images in comparison with the rigid motion and deformation modeling by the TPS method. 

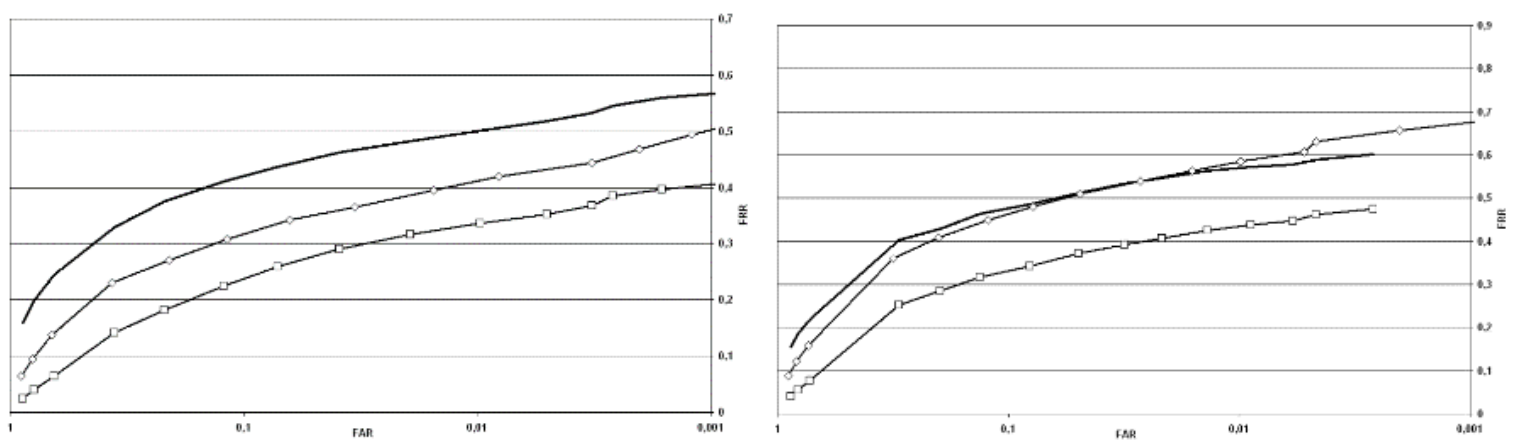

- optimal movement

$-\checkmark-$ accounting for deformations (model TPS)

$-\square-$ accounting for deformations (elastic model)

Figure 6. DET of the direct overlap factor (FVC2002DB1) and (FVC2004DB1)

In this study devoted to methods of analysis and synthesis of deformations of fingerprints. To determine the deformation structure, an analysis was made of the numerical solutions of (9) on publicly available fingerprint databases. Since (8) is linear, its solutions (deformations) can be added and multiplied by a number. For deformations, operations of the scalar product and projection onto each other are also defined. Therefore, it is proposed to study the structure of deformations by linear expansion in terms of an "orthonormal" set of basis deformations [20].

Basic deformations can be calculated both from qualitative considerations, and through formal statistical analysis. Based on the expansion (13), an approximate linear model is developed. Coefficients $c_{i}$ are calculated by minimizing the discrepancy (9) and (10). As basic deformations, it is suggested to use the "principal" deformations obtained by the main component method on the reference fingerprint array [21, 22]. To calculate the main components, three FVC2002 databases were used, containing images from optical and capacitive fingerprint scanners [23, 24]. Each database contains 8 samples for 100 people, i.e. for the analysis of deformities, there are three sets of 2800 natural deformations of fingerprints in total $[25,26]$.

In Figure 7 shows the first four basic deformations obtained by the principal component method. They allow a physical interpretation (two types of rotation and two shifts) see Figure 8. Such deformations correspond to the subjective concept of the main ways of applying a finger to the scanner. In addition to the qualitative analysis of deformations, a number of formal criteria for the accuracy of the deformation approximation were considered, which showed a rather high accuracy of the linear deformation model.

The first 8-10 main components provide a sufficiently accurate approximation of the observed strains for any of the above accuracy criteria. At the same time, the main components obtained are practically independent of the fingerprint array chosen for analysis [27, 28]. Differences in the nature of the deformations are in the various observed distributions of the coefficients $c_{i}$ of the expansion (12). From the data obtained, in particular, the following conclusions were: The statistical properties of natural deformations are practically independent of the type of scanner model (cases a and b). Synthetic deformations obtained by methods known in the past differ in their characteristics (c). Also, the quantitative difference between the deformations in the rolling of a print and the deformation in electronic scanning (live scan) is established. Then deals with the application of the developed methods of analysis and synthesis of deformations to real problems of fingerprint identification: Synthesis of artificial deformations of fingerprints and synthesis of "not-deformed" fingerprint to increase the accuracy of fingerprint identification [29]. The creation of a "not-deformed" averaged image from several fingerprints is a special case of the synthesis of artificial deformation. Let there be $\mathrm{k}+1$ images: $\mathbf{I m}_{0}, \mathbf{I m}_{1}, \ldots . \mathbf{I m}_{k}$. Relative deformations $\mathbf{I m}_{i}$ in $\mathbf{I m}_{0}$ are denoted by $\mathbf{u}_{i}$. Two approaches to the synthesis of mean strain are considered. The first is to formally calculate the average displacement map $\mathbf{u}_{a v}$ :

The average image is determined as $\mathbf{I m}_{a v}=\mathbf{u}_{a v}\left(\mathbf{I m}_{0}\right)$. The second approach is to calculate the state of the print, in which the intrinsic energy of the deformations to the images from the collection was minimal: The average for this condition is defined as $\mathbf{I m}_{\mathbf{m i n}}=\mathbf{u}_{\mathbf{m i n}}\left(\mathbf{I m}_{\mathbf{0}}\right)$. The resulting "not-deformed" image is used to increase the accuracy of identification. When registering on the basis of several fingerprints, the average state is calculated shown Figure 9. The solution (12) depends only on the mutual arrangement of control points in various applications of the finger. Therefore, the not-deformed state can be calculated not only for the image of the fingerprint, but also for the fingerprint template. Thus, the synthesis of the "not-deformed" state can be introduced into functioning automatic dactyloscopic verification and identification systems [30, 31]. 


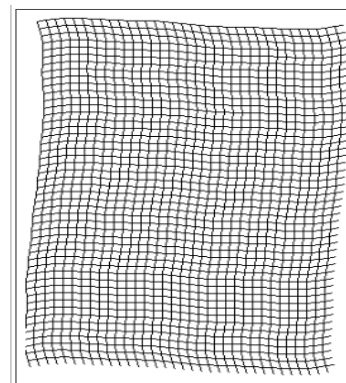

(a)

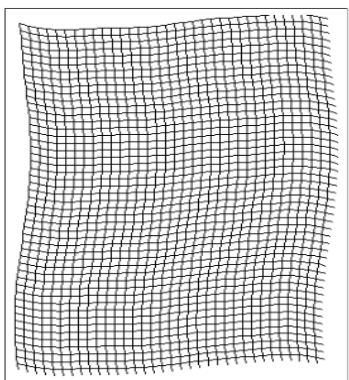

(b)

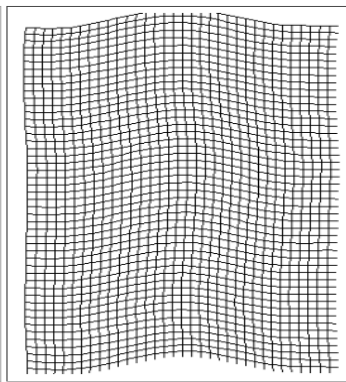

(c)

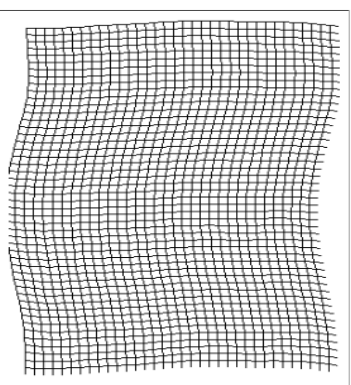

(d)

Figure 7. Map of the displacements of the main deformations obtained on different fingerprint arrays, (a), (b) deformation rotation, (c), (d) deformation shifts

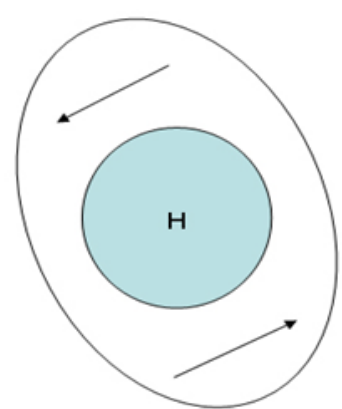

(a)

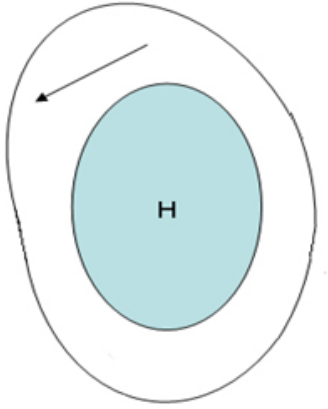

(b)

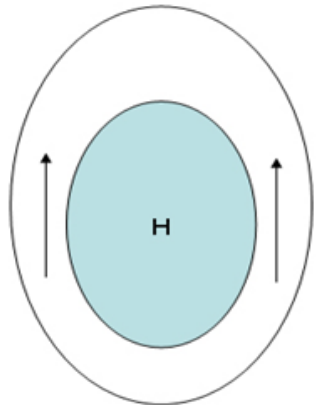

(c)

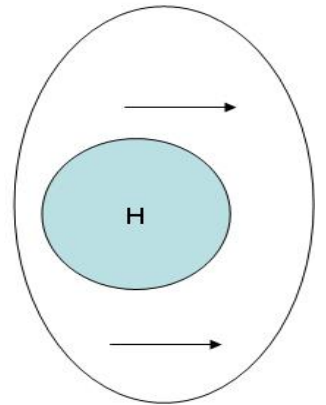

(d)

Figure 8. Schematic representation of the main deformations, "H" - relatively fixed area, the arrow indicates the main directions of displacement, (a), (b) deformation rotation, (c), (d) deformation shifts

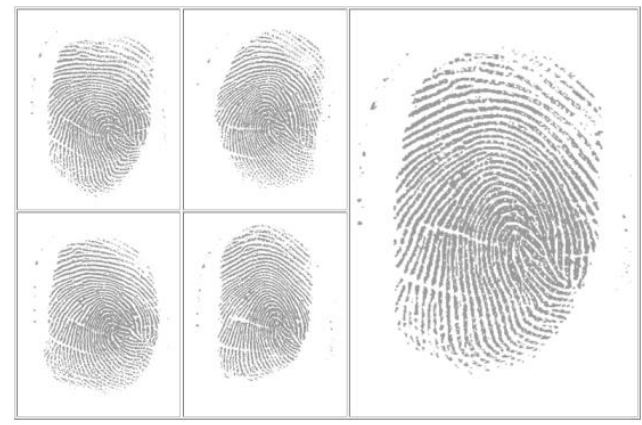

Figure 9. Averaged deformation fingerprint image $\left(\mathrm{Im}_{\min }\right)$

\section{CONCLUSION}

A model of elastic fingerprint deformations based on the picture of the print as an anisotropic elastic material is suggested taking into consideration the heterogeneity of fingerprint mechanical properties in the sense of the local projection of the deformation direction in the local direction of the papillary line flows. An approximate methods and algorithms have been developed for fingerprint deformation calculations based on solution of elastic deformation equations that help the mechanical characteristics of fingerprints. Methods are built for the study and synthesis of fingerprint image deformations based on the expansion of deformations by major types of applied force (deformation movements and rotations). A feature system has been developed that characterizes the deformation structure. The composition of the fingerprint deformations was statistically and qualitatively analyzed for the various fingerprint databases. An information technology has been developed to synthesize artificial deformations with statistical characteristics of observed natural deformations. An information technology has been developed in order to synthesize a "non-deformed" fingerprint prototype for fingerprint recognition and testing systems. 


\section{REFERENCES}

[1] D. A. Reid, et al., "Soft biometrics for surveillance: an overview," in Handbook of statistics, vol. 31, pp. 327-352, 2013.

[2] M. S. Al-Ani and W. M. Al-Aloosi, "Biometrics fingerprint recognition using discrete cosine transform (DCT)," International Journal of Computer Applications, vol. 69, no. 6, pp. 44-48, 2013.

[3] F. A. Fernandez, et al., "Quality Measures in Biometric Systems," IEEE Security \& Privacy, vol. 10, no. 6, pp. 52-62, 2011.

[4] H. U. Jang and H. K. Lee, "Biometric-PKI authentication system using fingerprint minutiae," Journal of Computer and Communications, vol. 2, no. 4, pp. 25-30, 2014.

[5] M. M. Kwakye, et al., "Adoption of Biometric Fingerprint Identification as an Accessible, Secured form of ATM Transaction Authentication," International Journal of Advanced Computer Science and Applications, vol. 6, no. 10, pp. 253-265, 2015.

[6] I. G. Telleria, et al., "Low-Cost and Efficient Hardware Solution for Presentation Attack Detection in Fingerprint Biometrics Using Special Lighting Microscopes," IEEE Access, vol. 7, pp. 7184-7193, 2019.

[7] M. Joshi, et al., "Security vulnerabilities against fingerprint biometric system," arXiv: 1805.07116, 2018.

[8] A. S. Jubair, et al., "Scale Invariant Feature Transform Based Method for Objects Matching," in 2019 International Russian Automation Conference (RusAutoCon), pp. 1-5, 2019.

[9] H. I. Wahhab, "Clustering Method of Fingerprint Flow Map and Coherence," in 2019 International Conference on Industrial Engineering, Applications and Manufacturing (ICIEAM), pp. 1-5, 2019.

[10] C. Obimbo and W. Wang, "A Fuzzy Clustering Algorithm for Fingerprint Enhancement," in Proceedings of the International Conference on Security and Management (SAM), 2011.

[11] V. Singh and I. Elamvazuthi, "Fingerprint matching algorithm for poor quality images," The Journal of Engineering, vol. 2015, no. 4, pp. 149-153, 2015.

[12] N. Bhargava, et al., "Fingerprint recognition using minutia matching," International Journal of Computer Trends and Technology, vol. 3, no. 4, pp. 504-507, 2012.

[13] S. K. Abbas, et al., "Comparative study of SVM-based classification techniques for human facial recognition," International Journal of Civil Engineering and Technology, vol. 9, no. 12, pp. 881-889, 2018.

[14] K. Noor, et al., "Performances Enhancement of Fingerprint Recognition System Using Classifiers," IEEE Access, vol. 7, pp. 5760-5768, 2018.

[15] M. H. Abdulameer, et al., "Face recognition technique based on adaptive-opposition particle swarm optimization (AOPSO) and support vector machine (SVM)," ARPN Journal of Engineering and Applied Sciences, vol. 13, no. 6, pp. 2259-2266, 2018.

[16] C. Lin and A. Kumar, "Matching contactless and contact-based conventional fingerprint images for biometrics identification," IEEE Transactions on Image Processing, vol. 27, no. 4, pp. 2008-2021, 2018.

[17] H. S. Khalifa, et al., "Fingerprint Segmentation Approach for Human Identification," Applied Mathematics and Information Science, vol. 13, no. 4, pp. 515-521, 2019.

[18] V. Rekha, et al., "Dactyloscopy Based Gender Classification Using Machine Learning," in 2019 IEEE International Conference on System, Computation, Automation and Networking (ICSCAN), pp. 1-5, 2019.

[19] A. O. Akinyele, et al., "Fingerprint Verification System Using Combined Minutiae and Cross Correlation Based Matching," American Journal of Electrical and Computer Engineering, vol. 2, no. 2, pp. 16-26, 2018.

[20] I. Ito and A. Pižurica, "Three-Dimensional Block Matching Using Orthonormal Tree-Structured Haar Transform for Multichannel Images," Journal of Imaging, vol. 6, pp. 4-21, 2020.

[21] J. J. Engelsma, et al., "Universal 3D wearable fingerprint targets: advancing fingerprint reader evaluations," IEEE Transactions on Information Forensics and Security, vol. 13, no. 6, pp. 1564-1578, 2018.

[22] C. R. Chowdhury and B. Saha, "Efficient fingerprint matching based upon minutiae extraction," International Journal of Advanced Computer Research, vol. 5, no. 21, pp. 347-354, 2015.

[23] M. K. Mohsen, et al., "Achieving fixed-frequency beam scanning with a microstrip leaky-wave antenna using doublegap capacitor technique," IEEE Antennas and Wireless Propagation Letters, vol. 18, no. 7, pp. 1502-1506, 2019.

[24] C. J. Chen, et al., "A support vector machine approach for truncated fingerprint image detection from sweeping fingerprint sensors," Sensors, vol. 15, no. 4, pp. 7807-7822, 2015.

[25] F. Firdaus, N. A. Ahmad, and S. Sahibuddin, "Fingerprint indoor positioning based on user orientations and minimum computation time," TELKOMNIKA Telecommunication Computing Electronics and Control, vol. 17, no. 4, pp. 1740-1749, 2019.

[26] S. Yoon, et al., "Altered fingerprints: Analysis and detection," IEEE transactions on pattern analysis and machine intelligence, vol. 34, no. 3, pp. 451-464, 2012.

[27] H. I. Wahhab, "Novel Method of Fingerprint Segmentation," in 2019 International Russian Automation Conference (RusAutoCon), pp. 1-5, 2019

[28] S. Mohammedsayeemuddin, et al., "A simple and novel fingerprint image segmentation algorithm," in 2014 International Conference on Issues and Challenges in Intelligent Computing Techniques (ICICT), pp. 756-759, 2014.

[29] G. Ma and J. Liu, "Fingerprint Image Segmentation Method Based on Gray Vision Parameter," in 2010 WASE International Conference on Information Engineering, pp. 154-157, 2010.

[30] A. Dey, et al., "A new algorithm for finding minimum spanning trees with undirected neutrosophic graphs," Granular Computing, vol. 4, no. 1, pp. 63-69, 2019.

[31] M. K. Mohsen, et al., "Control Radiation Pattern for Half Width Microstrip Leaky Wave Antenna by using PIN Diodes," International Journal of Electrical and Computer Engineering, vol. 8, no. 5, pp. 2959-2966, 2018. 